\title{
ESTUDO COMPARATIVO DA MASSA ESPECÍFICA APARENTE E RETRATIBILIDADE DA MADEIRA DE PAU-BRASIL (Caesalpinia echinata LAM.) NATIVA E DE REFLORESTAMENTO ${ }^{1}$
}

\author{
Sinval dos Santos Marques², José Tarcísio da Silva Oliveira ${ }^{3}$, Juarez Benigno Paes ${ }^{3}$, Edenise Segala
} Alves $^{4}$, Aderbal Gomes da Silva ${ }^{3}$ e Nilton César Fiedler ${ }^{3}$

\begin{abstract}
RESUMO - Entre as propriedades físicas da madeira, a massa específica aparente e a retratibilidade são características importantes na avaliação da qualidade da madeira para ampla gama de uso. A massa específica aparente, ou densidade aparente, está relacionada a uma série de outras características, principalmente àquelas relacionadas às propriedades mecânicas e anatômicas da madeira. Do mesmo modo, a retratibilidade também é importante característica, pois avalia a estabilidade dimensional das madeiras, sendo de grande importância para aplicações em que a madeira não deve apresentar contrações excessivas durante sua utilização. Com base nisso, este estudo teve como objetivo comparar a massa específica aparente e a retratibilidade da madeira de pau-brasil, proveniente de áreas naturais e de reflorestamentos com 10, 15, 20, 26 e 30 anos. Tais avaliações foram realizadas segundo a metodologia preconizada pelo Método Brasileiro 26/1940 da Associação Brasileira de Normas Técnicas. Os resultados indicaram que houve variação na massa específica aparente nas diferentes idades, com os maiores valores para a madeira de 30 anos de idade, que foram próximos aos encontrados para amostras de madeira nativa. A retratibilidade volumétrica total também variou em função da idade, não apresentando grande diferença em relação à madeira proveniente de áreas naturais.
\end{abstract}

Palavras-chave: Pau-Brasil, Madeira e Propriedades físicas.

\section{SPECIFIC GRAVITY AND SHRINKAGE OF PERNAMBUCO WOOD (Caesalpinia echinata LAM.)}

\begin{abstract}
Among physical properties of the wood, specific gravity and shrinkage are the most important characteristics to evaluate the wood quality and indication to several uses. Apparent specific gravity or density is an important physical characteristic that is related with shrinkage and others characteristics asthe mechanical and anatomical properties of the wood. Shrinkage is also an important characteristic because it determines as wood dimensional stability which is an important property for several uses in which wood should not present excessive contraction when being used. Thus, the objective of this study was to compare the specific gravity and shrinkage of Pernambuco wood (Caesalpinia echinata) from natural areas and reforestation at the ages of 10, 15, 20, 25 and 30 years. This study followed the Brazilian Method 26/1940 of Brazilian Associations of Technical Standard. The results indicate that there was variation in the apparent specific gravity and dry density atthe different ages with the highest value found for the wood at 30thirty years of age which was similar to the ones found for samples of native wood. The wood shrinkage also presented variation in function of the age but it did do not show differences in relation to native wood.
\end{abstract}

Keywords: Pernambuco wood and Physical properties.

\footnotetext{
${ }^{1}$ Recebido em 26.11.2010 e aceito para publicação em 29.03.2012

${ }^{2}$ Fundação Ceciliano Abel de Almeida, FCAA, Brasil. E-mail: <sinvalcelulose@terra.com.br>.

${ }^{3}$ Universidade Federal do Espírito Santo, UFES, Brasil.E-mail: <jtsilva@npd.ufes.br>,<jbp2@uol.com.br>, <aderbalsilva@yahoo.com.br> e $<$ fiedler@pesquisador.cnpq.br>.

${ }^{4}$ Instituto de Botânica, IBT, Brasil. E-mail: <ealves@ibot.sp.gov.br>.
} 


\section{INTRODUÇÃO}

Atualmente, o setor de marchetaria (fabricantes de arcos) passa por um momento de busca de alternativas, pois a matéria-prima dessa área é a madeira de pau-brasil (Caesalpinia echinata Lam.) proveniente de poucas áreas de exploração legalizadas pelo Instituto Brasileiro do Meio Ambiente e dos Recursos Naturais Renováveis - IBAMA.

A alternativa para o setor seria o uso de madeira de pau-brasil oriunda de reflorestamento, porém, segundo o resumo do documento "Conservação e Manejo de Pau-Brasil Caesalpinia echinata - Plano de Ação” realizado em 1997, os marcheteiros acreditam que a madeira oriunda de reflorestamento não tenha a mesma densidade, propagação de som, elasticidade, cor, textura, entre outras, para substituir a madeira proveniente de áreas nativas (PERSPECTIVAS III, 2009).

Na fabricação de arcos, a densidade é um dos requisitos adotados na seleção da madeira, pois, de acordo com essa propriedade, a madeira terá uso específico para confecção de arco para violão cello, contrabaixo ou violino.

Segundo Klock (2000), o aprimoramento no emprego de novas tecnologias para transformação e uso racional da madeira na geração de novos produtos requer o conhecimento adequado de suas características e comportamento como matéria-prima. Para tanto, as propriedades físicas da madeira, tendo como destaque a densidade ou massa específica aparente e a retratibilidade, são uma das mais estudadas pela facilidade e rapidez de seus resultados e a relação com outras propriedades e características tecnológicas da madeira. Para a confecção de arcos de violino, tais características devem ser levadas em consideração.

A massa específica aparente reflete a quantidade de matéria lenhosa por unidade de volume, ou do volume de espaços vazios em dada madeira (KOLLMANN; CÔTÉ, 1968; VALE et al., 1992). É uma das propriedades mais importantes da madeira para os mais diversos fins, visto que sua variação afeta a resistência mecânica e, muitas vezes, a variação dimensional da madeira. Gonçalves et al. (2009) afirmaram que até o momento poucos são os trabalhos que relacionam todas as características exigidas pelo mercado consumidor às propriedades inerentes à madeira, como densidade. Esta, por ser a mais importante e a que melhor se relaciona com as demais propriedades da madeira, é a característica mais utilizada em pesquisas relacionadas à qualidade da madeira, podendo limitar seu uso (VALE et al., 1992; TRUGILHO etal., 1996; LATORRACA;ALBUQUERQUE, 2000; CRUZ et al., 2003). Está relacionada também com a anatomia da madeira, uma vez que é reflexo das dimensões das fibras ou traqueídeos (comprimento, largura e espessura da parede e do diâmetro do lume) (PANSHIN; De ZEEUW, 1980).

A variação da densidade da madeira de diversas espécies florestais tem sido relacionada com as variações ambientais, como as variáveis geográficas e a precipitação (ZOBEL; van BUIJTENEN, 1989; WIEMAN; WILLIANSON, 2002), que também afetam o desenvolvimento das árvores.

A retratibilidade é, de acordo com Oliveira (1998), definida como uma forma de variação dimensional da madeira quando há alteração no seu teor de umidade, resultando na movimentação (inchamento e contração) pela perda de água abaixo do ponto de saturação das fibras.

O fator de anisotropia é o resultado da razão entre as contrações tangencial e radial, que representam o comportamento da madeira em relação à secagem, indicando maior ou menor propensão das peças em empenar ou rachar. Verifica-se que a retratibilidade tangencial é maior que a radial, enquanto a retratibilidade longitudinal é praticamente desprezível (OLIVEIRA, 1998).

Segundo Oliveira (1988), os valores obtidos para o fator de anisotropia podem variar de 1,3 para madeira de elevada estabilidade dimensional até para valores superiores a 3,0, comumente encontrados em algumas madeiras de eucalipto, que se caracterizam por elevada instabilidade dimensional.

Em face das buscas por novas alternativas, este estudo visou comparar a massa específica aparente e a retratibilidade de amostras de pau-brasil oriundas de reflorestamento de diferentes idades com amostras provenientes de populações naturais do Bioma Mata Atlântica.

\section{MATERIAL E MÉTODOS}

\subsection{Localização e caracterização do material de coleta}

A madeira para este estudo foi obtida na área de reflorestamento de pau-brasil (Caesalpinia echinata Lam.) com espaçamento de 2,0 x 2,0 m. As coordenadas 
geográficas foram 1954’21,6" de Latitude Sul e 40¹8'02,3" de Longitude Oeste, de acordo com o "South American Datum 1969” (SAD 69), de propriedade da Empresa Horst John Cia Ltda., localizada em Santa Rosa, Distrito de Aracruz, ES. Para o estudo foram colhidas 25 árvores nas idades de 10, 15, 20, 25 e 30 anos, sendo cinco de cada idade.

Os tipos de solo predominantes na área são Latossolo Vermelho-Amarelo distrófico coeso (LVAd), algumas manchas de Podzólico Vermelho-Amarelo abrupto (Pvad), também apresentando solo tipo Gley na área de várzea. O relevo da área reflorestada é suave ondulado.

A temperatura média anual da região varia entre 21,3 e $24,4{ }^{\circ} \mathrm{C}$. Os meses mais quentes são janeiro e fevereiro, com a temperatura caindo gradativamente até atingir a mínima média mensal nos meses de julho e agosto. A precipitação média anual é de 1.200 mm.

As amostras dos indivíduos nativos foram obtidas através de doação do IBAMA e provenientes de um lote de madeira apreendida no Estado do Espírito Santo, procedente de uma área do bioma natural de Mata Atlântica no Estado da Bahia.

\subsection{Determinação da massa específica aparente e retratibilidade}

Os ensaios físicos foram realizados de acordo com o preconizado pelo Método Brasileiro 26 - da Associação Brasileira de Normas Técnicas - MB-26 ABNT (1940), com algumas alterações no que se refere à determinação do volume pelo método hidrostático, com substituição do mercúrio pela água nas amostras saturadas.

Para o estudo da massa específica aparente (básica e anidra) e da retratibilidade da madeira proveniente de reflorestamento, foram coletadas duas toras de 1,2 m de comprimento a partir da base de cada árvore, totalizando 50 toras para os cinco tratamentos. As toras foram transportadas para o Laboratório de Ciência da Madeira (LCM), do Núcleo de Estudos e de Difusão de Tecnologia em Floresta, Recursos Hídricos e Agricultura Sustentável (NEDTEC), do Centro de Ciências Agrárias (CCA) da Universidade Federal do Espírito Santo (UFES), localizado no Município de Jerônimo Monteiro, ES, onde foram preparados os corpos-de-prova dos ensaios.

Da madeira oriunda de reflorestamento foram retirados 20 corpos-de-prova nas dimensões de $2,0 \times 2,0 \times 3,0 \mathrm{~cm}$ para cada tratamento, com a maior dimensão na direção axial, perfazendo um total de 100 corpos-de-prova. Para madeira nativa também foram preparados 20 corpos-de-prova, a partir de cinco peças selecionadas do lote recebido do IBAMA; com retirada de quatro corpos-de-prova por peça, com as mesmas dimensões anteriormente descritas.

Para determinação da massa específica básica, as amostras foram saturadas com água em dessecador, com aplicação de vácuo intermitente até que os seus teores de umidade fossem elevados acima do ponto de saturação das fibras. Determinou-se o volume saturado pelo método de deslocamento em água (VITAL, 1984), e as amostras foram secas em estufa, mantida a $103 \pm 2{ }^{\circ} \mathrm{C}$, até atingirem massas constantes e pesadas em uma balança de 0,01 g de precisão, sendo a densidade básica das madeiras obtida pelo método descrito por Vital (1984) (equação 1). Já a densidade anidra foi determinada ao se dividir a massa de matéria seca pelo volume de matéria seca em estufa, mantida nas condições anteriormente descritas. Para a determinação da retratibilidade, utilizou-se um Palmer com precisão de 0,001 mm. As dimensões das amostras foram medidas nas condições saturada e secas em estufa $\left(103 \pm 2{ }^{\circ} \mathrm{C}\right)$. Mediram-se as dimensões das amostras nas direções radial, tangencial e longitudinal, e calcularam-se as retratibilidades volumétrica, radial e tangencial e o coeficiente de anisotropia.

\subsection{Análise e avaliação dos resultados}

Os parâmetros avaliados foram analisados em função da média, desvio-padrão e coeficiente de variação. Além dessas análises, os valores de massa específica aparente (básica e anidra) $\left(\mathrm{g} \mathrm{cm}^{-3}\right)$, retratibilidade (radial, tangencial e volumétrica) (\%) e coeficiente de anisotropia foram analisados estatisticamente, para a verificação de ocorrência de diferenças entre os tratamentos. Depois da realização da ANOVA e verificada a significância do F, empregou-se o teste de Tukey a 5\% de significância, para verificação da ocorrência de diferença estatística entre os tratamentos.

\section{RESULTADOS}

Os valores médios encontrados de massa específica aparente, retratibilidade e coeficiente de anisotropia das madeiras de pau-brasil (Caesalpinia echinata Lam.) provenientes de reflorestamento e de madeira nativa encontram-se na Tabela 1.

Revista Árvore, Viçosa-MG, v.36, n.2, p.373-380, 2012 
Tabela 1 - Valores médios da massa específica aparente e retratibilidade da madeira de pau-brasil (Caesalpinia echinata) de diferentes idades e nativa.

Table 1 - Mean values of density and shrinkage of Caesalpinia echinata wood atdifferent ages and native wood.

\begin{tabular}{|c|c|c|c|c|c|c|}
\hline \multirow{2}{*}{$\begin{array}{l}\text { Idade } \\
\text { (Anos) }\end{array}$} & \multicolumn{2}{|c|}{$\begin{array}{c}\text { Massa específica } \\
\left(\mathrm{g} / \mathrm{cm}^{3}\right)\end{array}$} & \multicolumn{3}{|c|}{$\begin{array}{c}\text { Retratibilidade } \\
(\%)\end{array}$} & \multirow{2}{*}{$\begin{array}{c}\text { Coeficiente de } \\
\text { anisotropia } \\
\text { (T/R) }\end{array}$} \\
\hline & Básica & Anidra & Radial & Tangencial & Volumétrica & \\
\hline 10 & $\begin{array}{c}0,71 \mathrm{~d} \\
(0,06)(8,21)^{*}\end{array}$ & $\begin{array}{c}0,82 \mathrm{c} \\
(0,07)(8,66) \\
\end{array}$ & $\begin{array}{c}5,68 \mathrm{~b} \\
(0,58)(10,16) \\
\end{array}$ & $\begin{array}{c}9,23 \mathrm{bc} \\
(0,71)(7,66)\end{array}$ & $\begin{array}{c}15,17 \mathrm{~b} \\
(0,59)(3,92) \\
\end{array}$ & $\begin{array}{c}1,62 \mathrm{a} \\
(0,17)(10,33) \\
\end{array}$ \\
\hline 15 & $\begin{array}{c}0,77 \mathrm{c} \\
(0,04)(4,57)\end{array}$ & $\begin{array}{c}0,90 \mathrm{~b} \\
(0,04)(4,96)\end{array}$ & $\begin{array}{c}5,90 \mathrm{ab} \\
(0,21)(3,62)\end{array}$ & $\begin{array}{c}10,26 \mathrm{a} \\
(0,71)(6,90)\end{array}$ & $\begin{array}{c}16,24 \mathrm{ab} \\
(0,70)(4,29)\end{array}$ & $\begin{array}{c}1,66 \mathrm{a} \\
(0,09)(5,18)\end{array}$ \\
\hline 20 & $\begin{array}{c}0,81 \mathrm{~b} \\
(0,02)(2,11)\end{array}$ & $\begin{array}{c}0,95 \mathrm{a} \\
(0,03)(2,63)\end{array}$ & $\begin{array}{c}6,39 \mathrm{a} \\
(0,32)(5,04)\end{array}$ & $\begin{array}{c}10,64 \mathrm{a} \\
(1,04)(9,74)\end{array}$ & $\begin{array}{c}17,28 \mathrm{a} \\
(1,15)(6,67)\end{array}$ & $\begin{array}{c}1,62 \mathrm{a} \\
(0,17)(10,38)\end{array}$ \\
\hline 25 & $\begin{array}{c}0,76 \mathrm{c} \\
(0,02)(2,56)\end{array}$ & $\begin{array}{c}0,87 \mathrm{~b} \\
(0,02)(2,44)\end{array}$ & $\begin{array}{c}6,16 \mathrm{ab} \\
(0,97)(15,83)\end{array}$ & $\begin{array}{c}8,62 \mathrm{~b} \\
(0,78)(9,11)\end{array}$ & $\begin{array}{c}14,97 \mathrm{bc} \\
(1,23)(8,21)\end{array}$ & $\begin{array}{c}1,38 \mathrm{~b} \\
(0,16)(11,28)\end{array}$ \\
\hline 30 & $\begin{array}{c}0,86 \mathrm{a} \\
(0,02)(2,81)\end{array}$ & $\begin{array}{c}0,98 \mathrm{a} \\
(0,02)(1,82)\end{array}$ & $\begin{array}{c}5,03 \mathrm{c} \\
(1,19)(23,65)\end{array}$ & $\begin{array}{c}8,62 \mathrm{~b} \\
(1,70)(19,72)\end{array}$ & $\begin{array}{c}13,51 \mathrm{~d} \\
(3,03)(22,44)\end{array}$ & $\begin{array}{c}1,81 \mathrm{a} \\
(0,21)(11,84) \\
\end{array}$ \\
\hline Nativa & $\begin{array}{c}0,86 \mathrm{a} \\
(0,06)(6,90)\end{array}$ & $\begin{array}{c}0,98 \mathrm{a} \\
(0,07)(6,94)\end{array}$ & $\begin{array}{c}4,68 \mathrm{c} \\
(0,48)(10,24)\end{array}$ & $\begin{array}{c}8,46 \mathrm{~b} \\
(1,14)(13,43)\end{array}$ & $\begin{array}{c}14,19 \mathrm{~cd} \\
(1,60)(11,24)\end{array}$ & $\begin{array}{c}1,81 \mathrm{a} \\
(0,20)(10,79)\end{array}$ \\
\hline
\end{tabular}

Médias seguidas de mesma letra, dentro de cada coluna, não diferem entre si, pelo teste de Tukey a 5\% de significância. * Valores entre parênteses são desvio-padrão (em g/cm³ e \%) e coeficiente de variação (em \%), respectivamente.

Nota-se que a densidade básica da madeira oriunda de reflorestamento com idade de 30 anos não apresentou diferença estatística com relação à madeira nativa, tendo ambas mostrado densidade básica média de 0,86 g. $\mathrm{cm}^{-3}$. Esse valor de densidade pode ser considerado elevado. Seguindo a mesma tendência, a densidade anidra também não apresentou diferença estatística para a madeira de 30 anos e aquela nativa (Tabela 1).

O valor médio da densidade básica para a madeira de reflorestamento com 25 anos foi de $0,76 \mathrm{gcm}^{-3}$. Tendo esse valor sido semelhante ao da madeira de 15 anos e inferior ao encontrado para a madeira de 20 anos, tanto para a densidade básica quanto também para a anidra. O aumento da densidade com a idade da árvore é também encontrado em madeiras de outras espécies de dicotiledôneas. Silva et al. (2004) estudaram a influência da idade na massa específica aparente da madeira de Eucalyptus grandis de 10, 14, 20 e 25 anos. Verificaram que a madeira de 10 anos apresentou os menores valores de massa específica, seguida da madeira de 14 anos. Os maiores valores de massa específica foram encontrados na madeira de 20 anos, seguida da de 25.

A contração linear, na direção radial da madeira de 30 anos, não variou significativamente quando comparada com a da madeira nativa. As idades de 25,
15 e 10 anos foram estatisticamente semelhantes, tendo contração superior à da nativa. A madeira com 20 anos foi a que teve a maior contração linear na direção radial.

Na contração linear na direção tangencial, as idades de 20 e 15 anos apresentaram os maiores valores, diferindo estatisticamente dos demais, que tiveram média semelhante à da madeira nativa.

A contração volumétrica das madeiras provenientes das idades de 10 (15,16\%), 25 (14,97\%) e 30 anos (13,47\%) foi estatisticamente igual à da nativa (14,19\%), tendo a de idade de 30 anos o menor valor, inferior à da nativa. As árvores com 15 e 20 anos produzem madeira com contrações tangenciais iguais estatisticamente, sendo mais elevadas que os valores encontrados naquelas das demais idades.

Para o coeficiente de anisotropia, somente a madeira de 25 anos se destacou por apresentar diferença estatisticamente significativa entre as demais, provenientes de reflorestamento, e a madeira nativa, com um valor de 1,38. As demais idades tiveram valores variando de 1,61 a 1,81 .

Uma ilustração do comportamento da massa específica aparente da madeira de pau-brasil proveniente de reflorestamento e nativa está apresentada na Figura 1.

Os parâmetros da retratibilidade para a madeira de pau-brasil proveniente de reflorestamento e nativa estão apresentados na Figura 2. 


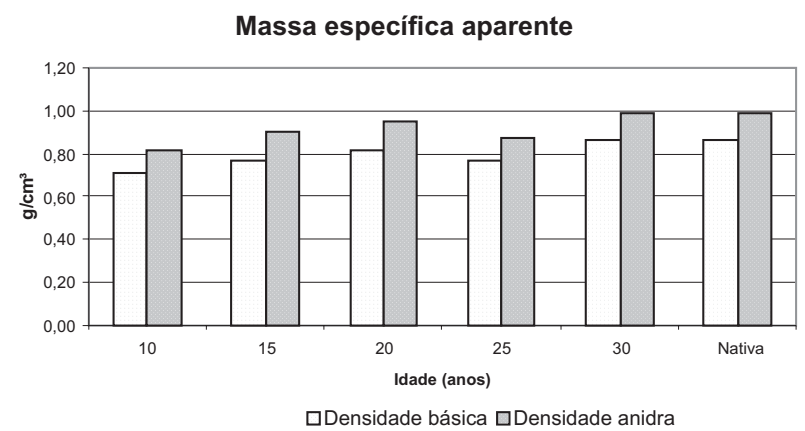

Figura 1 - Representação gráfica da massa especifica aparente para a madeira de pau-brasil (Caesalpinia echinata) de diferentes idades e nativa.

Figure 1 - Graphic representation of the density to Caesalpinia echinata wood in several ages and native wood.

Retratibilidade

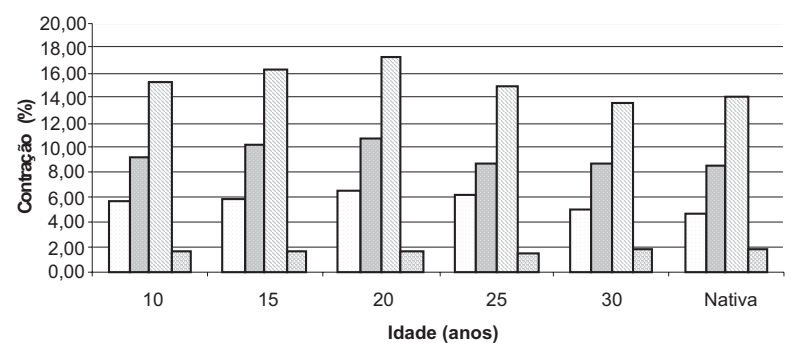

पC. Radial

口C. Tangencial $\quad \square$ C. Volumétrica

DFator anistrópico

Figura 2-Representação gráfica dos parâmetros de retratibilidade para a madeira de pau-brasil (Caesalpinia echinata) de diferentes idades e nativa.

Figure 2-Graphic representation of retratibility parameters to Caesalpinia echinata wood in different ages and native wood.

\section{DISCUSSÃO}

Os resultados indicaram diferença significativa entres as madeiras de 25 e 30 anos, com relação à densidade, e não conferem com os da literatura (Tabela 1), pois, segundo Giordano (1961), Boyd (1967) e Harris (1969), madeiras em idades mais avançadas aumentam o comprimento e espessura da parede da fibra, a porcentagem da madeira madura (cerne), a porcentagem de extrativos e, consequentemente, a densidade básica. Nesse caso, acredita-se que as árvores de 30 anos possuem maior quantidade de lenho adulto em relação àquelas de 25.
Lawton (1984) relatou que, numa mesma zona geográfica, as variações na densidade da madeira são produtos das variações microclimáticas como ocorrência e intensidade de vento e topográficas como grau de inclinação do terreno, entre outros fatores. A densidade da madeira varia dentro da mesma espécie entre limites mais ou menos próximos. É influenciada por fatores ambientais como fertilidade do solo e disponibilidade de água (PEREIRA, 1937).

Longui (2005), em estudo comparativo entre madeiras de pau-brasil, ipê (Tabebuia sp.) e maçaranduba (Manilkara sp.) para fabricação de arcos para instrumento de corda, adotou a densidade e velocidade de propagação do som para a seleção das varetas. Esse mesmo pesquisador mencionou que, quanto maior a densidade, maior a resistência do arco, além de obter o peso final ideal com menor quantidade de madeira e excelente acabamento. Com isso, os efeitos da idade das árvores podem ter sido influenciados pelo sítio em que as árvores foram plantadas. Isso porque, durante a análise dos pontos de coleta, observou-se que as árvores de 25 anos estavam próximas da área de baixada do talhão e no caminho de escoamento da água oriunda das precipitações pluviométricas.

No setor de marchetaria, é comum classificar a madeira, de acordo com sua qualidade, em "top" (profissional, semiprofissional e estudante), conforme a densidade e propagação da velocidade do som medido pelo uso do aparelho “G. Lucchi Elasticity Tester”, descrito por Longui (2005). No caso da Empresa Horst John Cia Ltda., tais varetas darão origens a arcos denominados ouro, prata, cobre e estudante, respectivamente.

Longui (2005), ao comparar a madeira de pau-brasil com as de outras espécies nativas empregadas na confecção de arcos para instrumentos de corda, por meio de ensaios destrutivos, concluiu que varetas com densidade aparente a $12 \%$ de umidade, que apresentaram valores entre $0,95 \mathrm{~g} \mathrm{~cm}^{-3}$ e $1,05 \mathrm{~g} \mathrm{~cm}^{-3}$, foram consideradas ideais para produzir arcos de qualidade.

Apenas as densidades das madeiras provenientes de árvores de 30 anos e a de mata nativa apresentaram densidades próximas daquelas recomendadas por Longui (2005).

Revista Árvore, Viçosa-MG, v.36, n.2, p.373-380, 2012 
Pimentel Gomes (2000) considerou os coeficientes de variação como baixos, quando inferiores a $10 \%$; médios, quando de 10 a 20\%; altos, quando entre 20 e $30 \%$; e muito altos, quando superiores a $30 \%$. Os coeficientes de variação da densidade básica e anidra variaram de 8,61 a 8,66\%, considerados baixos, validando os números de repetições utilizados e, consequentemente, a maior homogeneidade dessa madeira para a propriedade analisada.

De acordo com Klitzke (2007), a avaliação da qualidade da madeira segundo os coeficientes de anisotropia para as idades de 10,15, 20 e 30 anos classificou-a como madeira de média estabilidade dimensional, com a madeira de 25 anos podendo ser considerada muito estável dimensionalmente.

De forma geral, a idade de 25 é menos propensa a defeitos causados pela retratibilidade, em comparação com as demais madeiras.

As informações relacionadas a higroscopicidade e retratibilidade são importantes para estabelecer programas de secagem da madeira para garantir a qualidade do arco.

Outro ponto a ser destacado é quanto à estabilidade do próprio arco. Como sua construção é artesanal, a estabilidade dimensional afeta a qualidade do arco, pois no ato da construção são realizados orifícios na cabeça do arco e na extremidade oposta, para inserir e prender a crina, enquanto na extremidade oposta à cabeça é colocado o conjunto de talão e parafuso, que forma um dispositivo com a função de tensionar o arco. Caso haja contração volumétrica alta ou grande variação na retratibilidade radial e tangencial, isso pode vir a afetar a qualidade do som e até mesmo o número de paradas para o tensionamento da crina.

Oliveira (1988) e Kollmann e Côté Junior (1968) afirmaram que, quase sempre, a massa específica se apresenta correlacionada com a retratibilidade, a trabalhabilidade, a impregnabilidade, a durabilidade natural e as várias propriedades mecânicas da madeira.

Segundo Vital e Trugilho (1997), a magnitude da variação dimensional é normalmente maior na madeira de maior massa específica, em razão da maior quantidade de madeira por unidade de volume; além disso, madeiras de maior massa específica para um mesmo teor de umidade contêm mais água na parede celular.
Segundo Skaar (1988), a retratibilidade volumétrica é proporcional à densidade básica da madeira, sendo a correlação desta maior em espécies de clima temperado do que em espécies tropicais.

A madeira de 30 anos de idade apresentou a maior média de densidade básica quando comparada com a de 10 anos. Esse parâmetro, quando correlacionado com a contração volumétrica para o mesmo tratamento, apresentou comportamento inverso. Tal resultado está em desacordo com o proposto por Kollmann e Côté Junior (1968), em que maior massa específica implica maior contração. Tal resultado pode ter sido influenciado diretamente pelas propriedades químicas, principalmente teor de extrativos e holocelulose, o que corrobora o fato de a massa específica não ser o único fator que possa explicar a instabilidade dimensional da madeira (Tabela 1).

O valor médio da densidade básica da madeira de 30 anos foi igual ao daquela oriunda de mata nativa, sendo acompanhada pela idade de 20 anos, e todas com densidade básica acima de $0,80 \mathrm{~g} \mathrm{~cm}^{-3}$. Notou-se ainda que a média da densidade básica em valores absolutos, em comparação com a nativa, segue a ordem crescente de 10, 15, 20, 25 e 30 anos.

Em estudo com híbrido clonal de Eucalyptus urophylla x E. grandis, Gonçalves (2005) encontrou maiores valores de retratibilidade em árvores mais velhas, em razão da sua maior densidade básica. Tal comportamento foi observado nas amostras de madeira nativa e nas de plantio de 30 anos. Segundo Marques e Martins (2002), de maneira geral, quanto menor a contração, mais estável a madeira, o que está de acordo com os valores apresentados na Figura 2, em que tanto a madeira nativa quanto aquela de 30 anos de idade podem ser caracterizadas como de menor estabilidade dimensional, em face dos menores valores de contração apresentados.

\section{CONCLUSÕES}

A madeira de pau-brasil com idade de 30 anos apresentou o mesmo valor de densidade da madeira oriunda de mata nativa, o que poderá atribuir a essa madeira os mesmos usos da madeira nativa.

Houve variação na massa específica aparente nas diferentes idades, com maiores valores desse índice para a madeira de 30 anos de idade.

A retratibilidade volumétrica total também variou entre as madeiras de reflorestamento em razão da idade. 
Os valores de retratibilidade total da madeira nativa foram semelhantes àqueles encontrados em madeiras de reflorestamento.

A madeira de 30 anos de idade é tão estável dimensionalmente quanto aquela oriunda de mata nativa, como mostrado pelos valores iguais do coeficiente de anisotropia.

\section{AGRADECIMENTOS}

À Empresa Horst John Cia. Ltda., pela doação das árvores de reflorestamento; e ao IBAMA-ES, pela doação da madeira nativa, o que possibilitou a realização deste trabalho.

\section{REFERÊNCIAS}

ASSOCIAÇÃO BRASILEIRA DE NORMAS TÉCNICAS - ABNT. Ensaios físicos e mecânicos de madeiras - Método Brasileiro. MB-26/1940. Rio de Janeiro: 1940. 16p.

BOYD, J. D. Efecct of plantation conditions on wood properties and utilization. In: FAO WORLD SYMPOSIUM ON MAN-MAD FOREST AND THEIR INDUSTRIAL IMPORTANCE, Camberra, 1967. Documents... Rome: FAO, 1967. v.1. p.789-821.

CRUZ, C. R.; LIMA, J. T.; MUNIZ, G. I. B. Variações dentro das árvores e entre clones das propriedades físicas e mecânicas da madeira de híbridos de Eucalyptus. Scientia Forestalis, n.64, p.33-37, 2003.

GIORDANO, G. Aptude au travail du bois d' Eucalyptus. In: CONFERÊNCIA MUNDIAL DO EUCALIPTO, 2, 1961, São Paulo. Relatórios e documentos... São Paulo: FAO, 1961. v.2, p.1138-1146.

GONÇALVES, F. G. Avaliação da qualidade da madeira de híbrido clonal Eucalyptus urophyllla $\times$ grandis para produtos sólidos. 2005. 167f. Dissertação (Mestrado em Produção Vegetal) - Universidade Federal do Espírito Santo, Centro de Ciências Agrárias, Alegre, 2005.

GONÇALVES, F. G. et al. Densidade e variação dimensional de um híbrido clonal de Eucalyptus urophylla x Eucalyptus grandis. Revista Árvore, v.33, n.2, p.277-288, 2009.
HARRIS, J. M. Mejoriamento genético de arboles para elevar la calidade de la madeira: oportunidades y ventajas practicas. In: WORLD CONSULTATION ON FOREST TREE BREEDING, 2., Washington, 1969. Proceedings. Rome: FAO, 1969. v.1. p.22.

KLITZKE, R. J. Secagem da madeira. In: OLIVEIRA, J. T. S.; FIEDLER, N. C.; NOGUEIRA, M. (Eds.). Tecnologias aplicadas ao setor madeireiro. Jerônimo Monteiro: Suprema, 2007. p.271-342.

KLOCK, U. Qualidade da madeira juvenil de Pinus maximinoi H. E. More. 2000. 291f. Tese (Doutorado em Ciências Florestais) Universidade Federal do Paraná, Curitiba, 2000.

KOLLMANN, F. F. P.; CÔTÉ JUNIOR, W. A. Principles of wood science and technology. Berlin: Springer-Verlag, 1968. v1. 592p.

LATORROCA, J. V.; ALBUQUERQUE, C. E. C. Efeito do rápido crescimento sobre as propriedades da madeira. Floresta e Ambiente, v.7, n.1, p.279-291, 2000.

LAWTON, R. O. Ecological constraints on wood density in a tropical montane rain forest.

American Journal of Botany, v.71, n.2, p.261-267, 1984.

LONGHI, E. L. Pau-brasil e outras espécies nativas empregadas na confecção de arcos para instrumentos de corda: um estudo comparativo. 2005. $75 \mathrm{f}$. Dissertação (Mestrado em Botânica) -

Universidade de São Paulo, Instituto de Botânica, São Paulo, 2005.

MARQUES, M. H. B.; MARTINS, V. A. Secagem da madeira. Brasília: LPF/IBAMA, 2002.

OLIVEIRA, J. T. S. Caracterização da madeira de eucalipto para a construção civil. 1998. 429f. Tese (Doutorado em Engenharia Civil) - Escola Politécnica, Universidade de São Paulo, São Paulo, 1998.

OLIVEIRA, J. T. S. Estudo das propriedades físicas e tecnológicas da madeira da pindaíba (Xylopia sericea St. Hill.). 1988. 106f. Dissertação (Mestrado em Ciências Florestais) - Universidade Federal de Viçosa, Viçosa, MG, 1988.

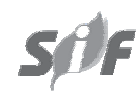

Revista Árvore, Viçosa-MG, v.36, n.2, p.373-380, 2012 
PANSHIN, A. J.; De ZEEUW, C. Textbook of wood technology. 3.ed. New York: McGraw Hill, 1980. 722p.

PEREIRA, J. A. As relações da estrutura anatômica do lenho com as propriedades físicas e mecânicas e os usos das madeiras.

Rodriguésia, v.11, p.337-341, 1937.

PERSPECTIVAS III: Um fórum para o paubrasil. Disponível em: <http:// www.sescsp.org.br/sesc/hotsites/paubrasil/cap9/ forum.htm>. Acesso em: 9 nov. de 2009.

PIMENTEL GOMES, F. Curso de estatística experimental. 14.ed. Piracicaba: Degaspari, 2000. 477p.

SILVA, J. C. et al. Influência da idade e da posição radial na massa específica aparente da madeira de Eucalyptus grandis Hill ex. Maiden. Floresta, v.34, n.1, p.13-22, 2004.

SKAAR, C. Wood-water relations. Berlin: Springer-Verlag, 1988. 283p.
TRUGILHO, F. P.; LIMA, J. T.; MENDES, L. M. Influência da idade nas características físicoquímicas e anatômicas da madeira de Eucalyptus saligna. Cerne, v.2, n.1, p.94-111, 1996.

VALE, A. T.; MARTINS, I. S.; ARAÚJO, W. O. Estudo da densidade básica de três espécies do cerrado. Revista Árvore, v.16, n.2, p.209-217, 1992.

VITAL, B. R. Métodos de determinação da densidade da madeira. Viçosa, MG: SIF, 1984. 21p. (Boletim Técnico, 1).

VITAL, B. R.; TRUGILHO, P. F. Variação dimensional e uso da Madeira de Eucaliptus. Informe Agropecuário, v.18, n.186, p.57-61, 1997.

WIEMAN, M.; WILLIANSON, G. B. Geografic variation in wood specific gravity: effects of latitude, temperature e precipitation. Wood and Fiber Science, v.34, n.1, p.96-107, 2002.

ZOBEL, B. J.; van BUIJTENEN, J. P. Wood variation: it's causes and control. Berlin: Springer Verlag, 1989. 363p. (Spring Series in Wood Science). 